\section{ORIGINAL RESEARCH}

A. Venmans

C.A.H. Klazen

P.N.M. Lohle

W.J. van Rooij

H.J.J. Verhaar

J. de Vries

W.P.Th.M. Mali

\title{
Percutaneous Vertebroplasty and Pulmonary Cement Embolism: Results from VERTOS II
}

BACKGROUND AND PURPOSE: The reported incidence of PCE during PV varies, depending on the sensitivity of diagnostic tests used. To assess the true incidence of PCE, we performed native chest CT during follow-up in a large proportion of patients from the VERTOS II trial.

MATERIALS AND METHODS: VERTOS II is a prospective multicenter randomized controlled trial comparing PV with conservative therapy in 202 patients. After a mean follow-up of 22 months (median, 21 months; range, 6-42 months), 54 of 78 patients (69\%) with 80 vertebrae treated with PV underwent native chest CT to detect possible PCE. The presence, location, number, and size of PCE were recorded. In addition, the presence of pulmonary parenchymal changes adjacent to PCE was noted. Possible risk factors for PCE, such as age, sex, number of treated vertebrae, cement volume per vertebra, and presence and location of perivertebral cement leakage, were evaluated.

RESULTS: PCE was detected in 14 of 54 patients $(26 \% ; 95 \% \mathrm{Cl}, 16 \%-39 \%)$. All patients were asymptomatic. Cement emboli were small and randomly distributed in peripheral small vessels. There were no reactive pulmonary changes. Cement leakage in the azygos vein was the only risk factor for the occurrence of PCE (OR, 43; 95\% Cl, 5-396).

CONCLUSIONS: Small and clinically silent PCE occurred in a quarter of patients treated with PV. Cement leakage into the azygos vein was the only risk factor. With time, these small cement emboli remained inert, without inflammatory pulmonary response. Standard postprocedural CT or chest radiographs are not necessary.

ABBREVIATIONS: $\mathrm{Cl}=$ confidence interval; $\mathrm{HU}=$ Hounsfield unit; OR = odds ratio; OVCF= osteoporotic vertebral compression fracture; PCE = pulmonary cement embolism; $\mathrm{PV}=$ percutaneous vertebroplasty; VERTOS = Percutaneous Vertebroplasty versus Conservative Therapy

C ement leakage commonly occurs during PV, with occurrence in observational studies varying from $0 \%$ to $23 \% .^{1-6}$ Occasionally, cement that has leaked into the veins may migrate into the lungs causing PCE. Although most PCEs remain asymptomatic, serious and even fatal sequelae have occasionally been reported. ${ }^{7,8}$ In most studies with a low incidence of PCE, "the occurrence of PCE" is defined as cement migration toward the lungs observed during fluoroscopy. ${ }^{9}$ In studies with standard postprocedural chest radiographs, the observed incidence is higher ${ }^{10,11}$; apparently, a substantial proportion of PCE remains undetected during fluoroscopy. In 1 study, the long-term effects of pulmonary cement deposits on the surrounding lung parenchyma are largely unknown. In this study, we used follow-up chest CT to assess the true incidence of the occurrence of PCE during fluoroscopy in a large patient cohort with osteoporotic vertebral compression fractures treated with PV. In addition, we evaluated the possible inflammatory response of cement deposits on the lung parenchyma.

Received February 5, 2010; accepted after revision March 9

From the Departments of Radiology (A.V., C.A.H.K., P.N.M.L., W.J.v.R.) and Medical Psychology (J.d.V.), St. Elisabeth Hospital, Tilburg, the Netherlands; and Departments of Geriatric Medicine (H.J.J.V.) and Radiology (W.P.Th.M.M.), University Medical Centre Utrecht, Utrecht, the Netherlands.

The sponsors of the study had no role in the study design, data collection, data analysis, data interpretation, writing of the report, or the decision to submit the paper for publication.

Please address correspondence to Alexander Venmans, MD, Department of Radiology, St. Elisabeth Ziekenhuis, Hilvarenbeekseweg 60, 5022 GC Tilburg, the Netherlands; e-mail: alexandervenmans@hotmail.com

DOI 10.3174/ajnr.A2127

\section{Materials and Methods}

\section{Patients}

The VERTOS II Trial ${ }^{12}$ was a pragmatic randomized controlled trial comparing PV and conservative therapy for OVCFs in 202 patients. The study protocol is described in detail elsewhere. ${ }^{12}$ In short, VERTOS II was conducted in 5 large teaching hospitals in the Netherlands and 1 in Belgium. The protocols of VERTOS II, including the present study, were approved by the institutional review boards at each participating center. Between October 2005 and June 2008, 202 patients were randomized for PV and conservative therapy. Ultimately, in 98 patients, PV was performed without clinical procedural complications. These 98 patients form the basis of the present study.

During a mean follow-up of 22 months (median, 21 months; range, $6-42$ months), 10 patients died and 6 refused to complete the protocol of VERTOS II. The remaining 82 patients were invited by telephone for a native CT of the treated vertebrae and chest to detect perivertebral cement leakage and PCE. Of these 82 patients, 24 declined participation and 4 patients could not be reached. Thus, 54 of 82 patients (69\%) had follow-up CT. CT was performed after a mean follow-up of 22 months (median, 21 months; range, 6-42 months). In these 54 patients, no cement migration had been visible on fluoroscopy during the procedure. There were 36 women $(67 \%)$ and 18 men $(33 \%)$ with a mean age of 74 years (median, 77 years; range, 53-88 years). These 54 patients had 80 OVCFs and were treated in 60 sessions. Thirty-nine patients were treated for $1 \mathrm{OVCF}$; 11 patients, for 2; and 4 patients, for 3 OVCFs in 1 session. During follow-up, 4 patients presented with a new OVCF and were treated again. One of these 4 patients had 2 additional PVs for 1 OVCF each time. The location of treated osteoporotic compression fractures in relation to PCE is shown in Fig 1. 


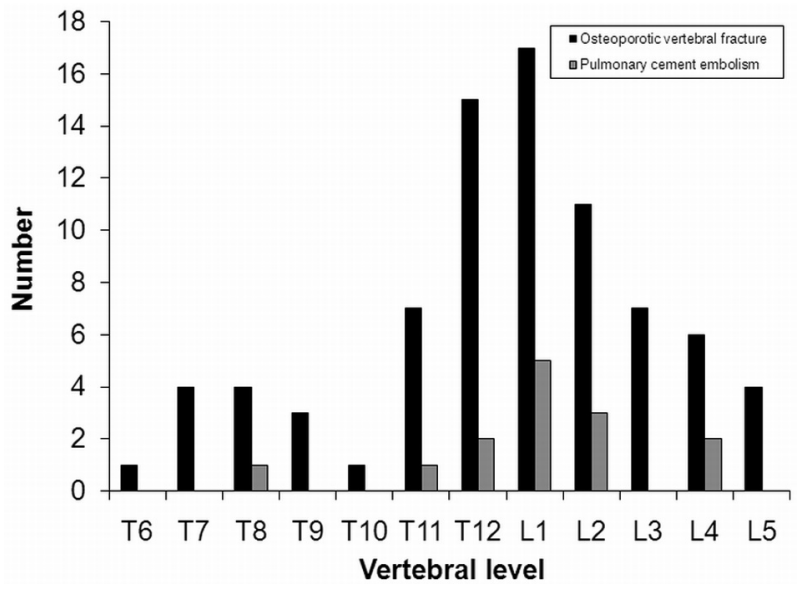

Fig 1. Location of treated osteoporotic compression fractures in relation to PCE.

\section{PV Technique}

PV was performed on a single or biplane angiographic unit under fluoroscopic guidance. After local infiltration anesthesia (lidocaine $1 \%$ ), needles were bilaterally transpedicularly inserted into the vertebral body. Polymethylmethacrylate bone cement (Osteo-Firm; Cook Medical, Bloomington, Indiana) was injected under continuous lateral fluoroscopy, alternating both pedicles by using 1-mL syringes. Injection was stopped whenever perivertebral cement migration was observed. Injection was resumed after a 15- to 20-second delay without changing needle position. The volume of injected cement in each treated vertebral body was recorded. Immediately after the procedure, a CT scan of the treated OVCF was obtained to evaluate perivertebral cement leakage.

\section{Follow-Up CT}

Follow-up CT was performed in 5 different hospitals with a multidetector spiral CT scanner with 16- or 64-detector arrays. Native CT of the chest and treated vertebrae was performed with a section thickness of $2 \mathrm{~mm}$. Two radiologists reviewed the follow-up CT scans. Differences were resolved by consensus. Cement-embolus detection was performed at a bone window (window width, $2400 \mathrm{HU}$; window level, $350 \mathrm{HU}$ ) and lung window (window width, $1500 \mathrm{HU}$; window level, $-700 \mathrm{HU})$. PCE should be located in the expected course of a pulmonary vessel with an attenuation of $>500 \mathrm{HU}$. To distinguish a calcified granuloma and a PCE, we made a comparison with an old chest radiograph or chest CT scan if possible.

\section{Perivertebral Cement Leakage on Postprocedural and Follow-Up CT}

Treated vertebrae were assigned to 3 location categories: T5-T10, T11-L2, and L3-L5. Perivertebral venous cement leakage was assessed from direct postprocedural CT and categorized as limited to the anterior external venous plexus, azygos vein, or inferior vena cava. "PCE" was defined as any high-attenuation lesion in the lungs, heart, or large vessels. In patients with PCE and multiple levels treated, we assumed the level with the most leakage to be the origin of PCE.

\section{Data Analysis}

The frequency of PCE was assessed per patient as a proportion, with 95\% CIs. Univariate logistic regression analysis was performed for the following possible risk factors for the occurrence of PCE: age, sex, number of treated vertebrae, cement volume injected per vertebra

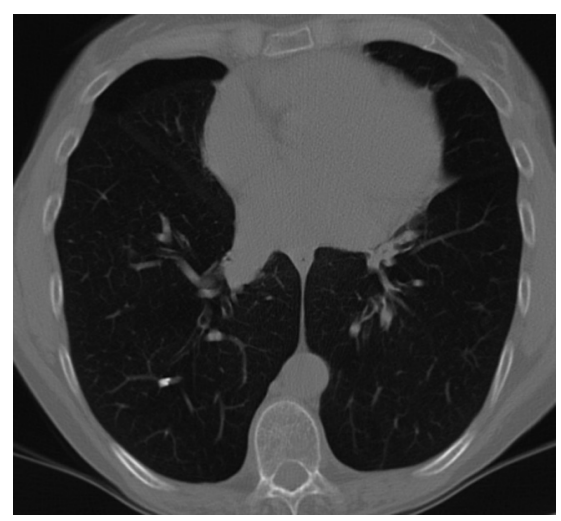

Fig 2. Native chest CT scan demonstrates a cement embolus in a peripheral right lower lobe pulmonary artery.

higher than the median, and presence and location of perivertebral venous cement leakage. The $\chi^{2}$ test was used to correlate PCE with the location of treated vertebrae. Statistics were performed with the Statistical Package for the Social Sciences, Version 15.0.1 (SPSS, Chicago, Illinois). The VERTOS II Trial is registered with clinicaltrials.gov, number NCT00232466.

\section{Results}

\section{Incidence and Characteristics of PCE on Native Follow- Up Chest CT}

After a median of 21 months of follow-up, PCE was detected in 14 of 54 patients (26\%; 95\% CI, 16\%-39\%). All patients were asymptomatic. An example of a PCE is presented in Fig 2.

The emboli varied in size between 1 and $12 \mathrm{~mm}$ and were randomly distributed in the periphery of the lungs. No cement depositions were observed in the heart and central pulmonary vessels. In the 14 patients with PCE, $6(43 \%)$ had a single cement embolus and 8 (57\%) had 2-35 cement depositions. With multiple PCEs, these emboli were randomly scattered in peripheral portions of both lungs. No patients showed reactive pulmonary parenchymal change associated with cement embolism.

\section{Cement Leakage after $P V$}

Venous cement leakage immediately after PV was observed on CT in 34 of 80 treated vertebrae (43\%); 23 cement deposits were into the anterior external venous plexus, 7 into the azygos vein, and 4 into the inferior caval vein.

\section{Statistical Analysis}

Cement leakage in the azygos vein was the only risk factor for the occurrence of PCE (OR, 43; 95\% CI, 5-396). Age, sex, number and location of treated vertebrae, and injected cement volume were not correlated with the occurrence of PCE.

\section{Discussion}

This study showed that during PV for OVCFs, clinically silent PCE occurs in a quarter of patients. Cement emboli were small and scattered in peripheral portions of the lungs without specific lobar distribution. There were no cement deposits in the heart and large vessels. Cement leakage in the azygos vein was the only risk factor for PCE. Remarkably, the volume of injected cement was not correlated with the occurrence of PCE. 
After a mean follow-up of almost 2 years, the cement emboli caused no structural parenchymal changes.

In a comparable study with the use of CT to detect PCE, Kim et $\mathrm{al}^{13}$ found a similar incidence in 75 patients undergoing PV for OVCFs, with cement leakage to the inferior caval vein as the only relevant risk factor. In studies that used only postprocedural chest radiographs for detection of PCE, the observed incidences were substantially lower. ${ }^{10,11}$ This is not surprising because small pulmonary cement deposits easily remain undetected on chest radiographs while they are readily apparent on CT. In 1 study, ${ }^{11}$ an incidence of $4.6 \%$ was reported after retrospectively reviewing postprocedural chest radiographs in $69 \mathrm{VP}$ sessions. In that study, all patients with cement emboli had multiple myeloma and remained asymptomatic. An association was found between PCE and paravertebral venous cement leakage but not between PCE and the number of vertebral bodies treated or the performance of kyphoplasty or vertebroplasty. In another study, ${ }^{10}$ the authors also retrospectively reviewed postprocedural chest radiographs, and when PCE was detected, they confirmed it with CT. In that study, 5 of 73 patients $(6.8 \%)$ had PCE. Four of these patients had osteoporotic compression fractures, and 1 had multiple myeloma. Venous leakage was not recognized during fluoroscopy in patients with PCE.

In the VERTOS II Trial, ${ }^{12}$ fluoroscopic venous cement migration to the lungs was detected and reported by the operator in only 1 patient (C.A.H. Klazen, unpublished data, 2010). This patient remained asymptomatic and stopped the trial 3 months later because of unrelated comorbidity. In no patients who were included in the present follow-up study was fluoroscopically visible cement migration toward the lungs reported by the operators. The findings of our study imply that with fluoroscopy, virtually all migration of small cement quantities remains undetected. Thus, when the operator notices cement leakage into anterior venous structures, careful observation of pulmonary symptoms is mandatory. Conversely, when a patient complains of pulmonary symptoms after PV, PCE should be excluded, even though venous cement migration was not seen.

As in previous studies, ${ }^{9-11}$ our study showed that cement emboli were scattered in peripheral portions of the lung without specific lobar distribution and no acute inflammatory pulmonary reaction. Our study also indicates that long-term, cement emboli do not cause structural changes of the pulmonary parenchyma. A previous study showed that PCE occurred infrequently and caused no pulmonary reaction on CT after 1 year. ${ }^{9}$

In our study, cement in the azygos vein on postprocedural $\mathrm{CT}$ of the treated vertebrae was the only risk factor for PCE. Analogously, other studies ${ }^{13,14}$ showed a statistically significant relation between PCE and cement leakage into the inferior caval vein.

Our study has several limitations. Our patient group was relatively small, and not all patients agreed to participate. Results were expressed on a per-patient basis, while some patients had multiple levels treated. Thus, in patients with PCE and multiple treated vertebrae, the level of leakage remained uncertain. However, we used postprocedural CT of the treated levels to indicate the most likely level of the origin of the leakage. Another limitation is that postprocedural chest $\mathrm{CT}$ was not routinely performed. Therefore, it is possible that small cement emboli might have been resolved or changed with time or had migrated. In addition, earlier but transient pulmonary changes cannot be excluded. On the other hand, strong points of the study were the use of CT for the detection of PCE and the long follow-up interval, which allowed a reliable assessment of PCE with time.

On the basis of our findings and other studies, in our opinion, standard CT or chest radiography after PV is not warranted in asymptomatic patients, not even when small quantities of cement have been observed migrating toward the lungs. Only in symptomatic patients should CT be performed to guide the appropriate therapy. Because cement emboli remain inert with time, follow-up CT is not necessary.

\section{Conclusions}

In the VERTOS II Trial, small and clinically silent PCE occurred in a quarter of patients treated with PV. Cement leakage into the azygos vein was the only risk factor. With time, these small cement emboli remained inert without inflammatory pulmonary response. Standard postprocedural CT or chest radiographs are not necessary.

\section{References}

1. Krueger A, Bliemel C, Zettl R, et al. Management of pulmonary cement embolism after percutaneous vertebroplasty and kyphoplasty: a systematic review of the literature. Eur Spine J 2009;18:1257-65

2. Jensen ME, Evans AJ, Mathis JM, et al. Percutaneous polymethylmethacrylate vertebroplasty in the treatment of osteoporotic vertebral body compression fractures: technical aspects. AJNR Am J Neuroradiol 1997;18:1897-904

3. Grados F, Depriester C, Cayrolle G, et al. Long-term observations of vertebral osteoporotic fractures treated by percutaneous vertebroplasty. Rheumatology (Oxford) 2000;39:1410-14

4. Zoarski GH, Snow P, Olan WJ, et al. Percutaneous vertebroplasty for osteoporotic compression fractures: quantitative prospective evaluation of long-term outcomes. J Vasc Interv Radiol 2002;13(2 pt 1):39-148

5. Legroux-Gerot I, Lormeau C, Boutry N, et al. Long-term follow-up of vertebral osteoporotic fractures treated by percutaneous vertebroplasty. Clin Rheumatol 2004;23:310-17

6. Diamond TH, Bryant C, Browne L, et al. Clinical outcomes after acute osteoporotic vertebral fractures: a 2-year non-randomised trial comparing percutaneous vertebroplasty with conservative therapy. Med J Aust 2006;184: 113-17

7. Chen HL, Wong CS, Ho ST, et al. A lethal pulmonary embolism during percutaneous vertebroplasty. Anesth Analg 2002;95:1060-62

8. Monticelli F, Meyer HJ, Tutsch-Bauer E. Fatal pulmonary cement embolism following percutaneous vertebroplasty (PVP). Forensic Sci Int 2005;149:35-38

9. Venmans A, Lohle PNM, van Rooij WJ, et al. Frequency and outcome of pulmonary polymethylmethacrylate embolism during percutaneous vertebroplasty. AJNR Am J Neuroradiol 2008;29:1983-85

10. Duran C, Sirvanci M, Aydooan M, et al. Pulmonary cement embolism: a complication of percutaneous vertebroplasty. Acta Radiol 2007;48:854-59

11. Choe DH, Marom EM, Ahrar K, et al. Pulmonary embolism of polymethyl methacrylate during percutaneous vertebroplasty and kyphoplasty. AJR Am J Roentgenol 2004;183:1097-102

12. Klazen CA, Verhaar HJ, Lampmann LE, et al. VERTOS II: percutaneous vertebroplasty versus conservative therapy in patients with painful osteoporotic vertebral compression fractures; rationale, objectives and design of a multicenter randomized controlled trial. Trials 2007;8:33

13. Kim JY, Lee JW, Park KW, et al. Pulmonary cement embolism after percutaneous vertebroplasty in osteoporotic vertebral compression fractures: incidence, characteristics, and risk factors. Radiology 2009;251:250-59

14. Barragán-Campos HM, Vallée JN, Lo D, et al. Percutaneous vertebroplasty for spinal metastases: complications. Radiology 2006;238:354-62 